Utah State University

DigitalCommons@USU

$1-1-1980$

\title{
Large Field-of-View Interferometers for Environmental Sensing
}

Doran J. Baker

Utah State University

Follow this and additional works at: https://digitalcommons.usu.edu/sdl_pubs

\section{Recommended Citation}

Baker, Doran J., "Large Field-of-View Interferometers for Environmental Sensing" (1980). Space Dynamics Lab Publications. Paper 9.

https://digitalcommons.usu.edu/sdl_pubs/9

This Article is brought to you for free and open access by the Space Dynamics Lab at DigitalCommons@USU. It has been accepted for inclusion in Space Dynamics Lab Publications by an authorized administrator of DigitalCommons@USU. For more information, please contact digitalcommons@usu.edu.

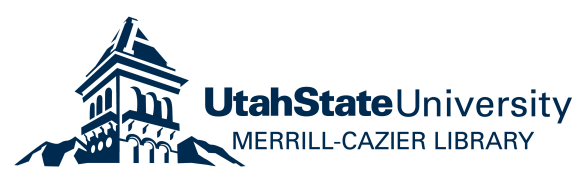




\section{Large field-of-view interferometers for environmental sensing}

\section{Doran J. Baker}

Utah State University

Dept. of Electrical Engineering

and Electro-Dynamics Laboratories

Logan, Utah 84322

\begin{abstract}
The interferometer-spectrometer is an exceptionally powerful instrument for remotely sensing optical emission spectra associated with the environment. However, when a conventional Michelson interferometer is operated at high spectral resolution, a very narrow viewing field results. For the sensing of emissions which have a significant spatial distribution, which is often the case for environmental species, optical compensation can be employed to open up the field of view. The resulting reduction in observation time for the compensated relative to the uncompensated interferometer is $R^{2} \theta^{4} / 4$. For a practical instrument using prism-type compensator elements, the viewing field can be extended to more than ten degrees full angle. Consequently, the observation time required to obtain a spectrum is reduced by more than two orders of magnitude.
\end{abstract}

Keywords: field of view; interferometer spectrometer; Fourier transform spectrometer.

Optical Engineering 19(4), 515-517 (July/August 1980)

\section{INTRODUCTION}

The basic relationship between resolving power $R$ and viewing field $\Omega$ for the classical Michelson symmetrical interferometer is

$\mathrm{R} \Omega=2 \pi$.

When this limitation is expressed in terms of the impact upon a practical spectrometer, the required collimation (in degrees off axis) of the incident light is ${ }^{1}$

$\theta=81\left(\mathrm{D}_{\mathrm{d}} / \mathrm{D}_{\mathrm{c}}\right) / \sqrt{\mathrm{R}}$,

where $D_{d} / D_{c}$ is the ratio of the detector diameter to the diameter of the collector.

The necessity to limit the field of view occurs because the amount of the optical retardation experienced by a light ray through the interferometer depends upon the angle of incidence (or obliqueness) of that ray. The effect can be demonstrated by referring to Fig. 1 which illustrates the interference pattern at the detector plane produced by a Michelson interferometer which is illuminated with a monochromatic source. The detector view is limited to the central image (ring) to prevent a loss of signal. The problem is that the size of this central image is much smaller for very oblique rays than it is for rays which are nearly on axis. These off-axis rays tend to "mush out" the resolution of the instrument. One solution is to eliminate the offending rays with a field stop. The alternative is to find some technique whereby the variation of path difference with incidence angle is cancelled out using optical compensation. This latter approach, called field compensation, requires additional optical components.

\section{FIELD-OF-VIEW COMPENSATION}

Consider the case where it is desired to obtain a spectrum of nitric oxide emissions near $1,875 \mathrm{~cm}^{-1}(\lambda 5.3 \mu \mathrm{m})$ to a resolution of $1 \mathrm{~cm}^{-1}$. The required collimation using Eq. (2) is 0.2 degrees. (The detector and collector sizes were taken as $1 / 2$ and $5 \mathrm{~cm}$ respectively.) This is a rather severe field-of-view limitation which, in turn, restricts the optical throughput (etendue) of the instrument when used to view the spatially extended atmospheric nitric oxide.

Original manuscript EO-102 received and accepted for publication March 13, 1980.

(c) Society of Photo-Optical Instrumentation Engineers

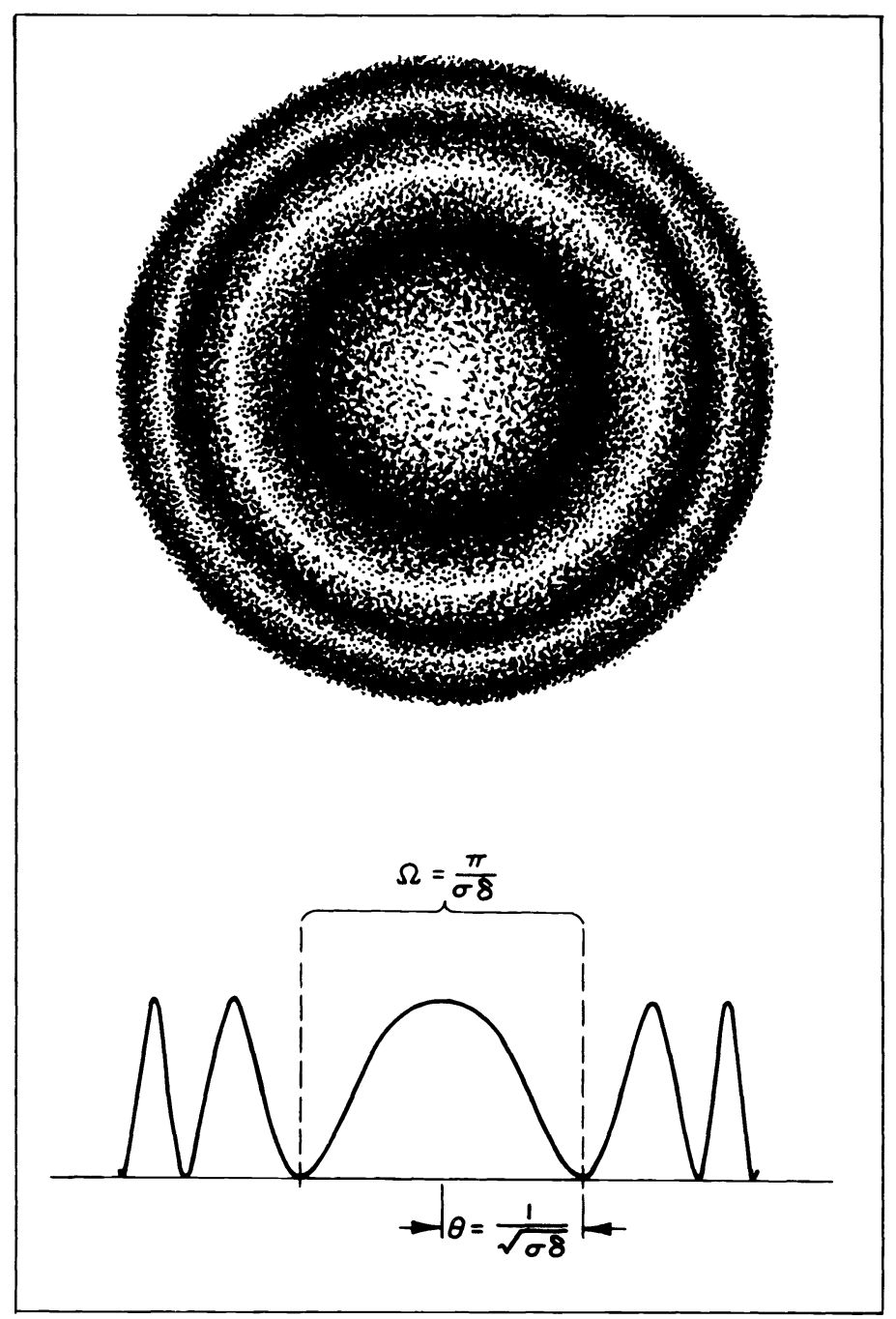

Fig. 1. Interference pattern at the detector plane of a Michelson interferometer which is illuminated by a monochromatic extended source. 
Many techniques have been suggested in the attempt to compensate for the image shift with entrance angle in an interferometer. ${ }^{1}$ One of the successful approaches was originally proposed by Bouchareine and Connes $^{2}$ and is illustrated in Fig. 2. Their idea was to insert a glass wedge

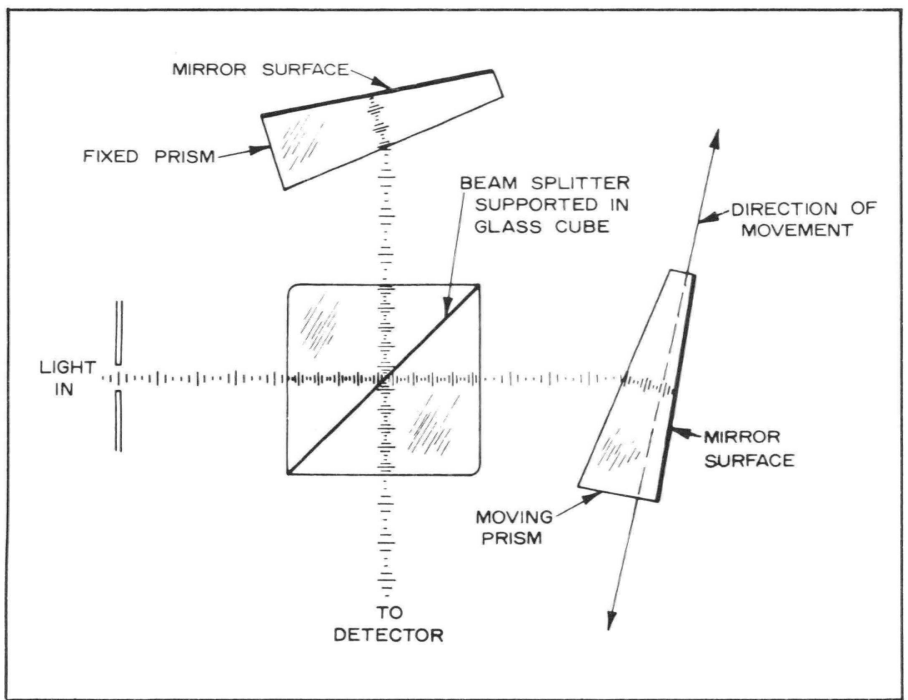

Fig. 2. Field compensation using wedge prisms.

or prism in each arm of the interferometer. Differential pathlength changes are then achieved by moving one of the prisms parallel with its apparent mirror position. More optical material is thereby inserted into one beam relative to the other to achieve an effective differential increase of pathlength. By selectively choosing the index of refraction of the material, the prism angles and the orientation of the prisms and mirrors, it is possible to obtain a first-order invariance of the interference pattern image at the detector to incidence angle of the incoming light.

Steed ${ }^{5}$ gives the necessary relationship under field-compensated conditions as follows

$\delta=2 \mathrm{~d}\left[\sin (\beta-\gamma)+\tan \alpha\left(1 / \mathrm{n}-1 / \mathrm{n}_{\mathrm{a}}\right)\right.$,

where $\delta$ is the retardation, $\mathrm{d}$ is the actual drive distance, $\beta$ is $\arcsin (\mathrm{n} \sin \alpha), \gamma$ is $\alpha-\arctan [(\mathrm{n}-1) / \mathrm{n} \tan \alpha], \alpha$ is the prism angle, $\mathrm{n}$ is the index of refraction and $n_{a}$ is the index of refraction at which the instrument is aligned.

Ware ${ }^{7}$ has pointed out that the entity

$\delta / 2 \mathrm{~d}=\mathrm{n}^{\prime}$

given in this compensation formula can be interpreted as an effective Michelson index of refraction $n^{\prime}$ for the field-compensated instrument. Therefore, the necessary drive to achieve a given maximum retardation (and hence spectral resolution) is

$\mathrm{d}=\delta / 2 \mathrm{n}^{\prime}$.

Since the effective index of refraction $n^{\prime}$ is less than unity, the necessary drive length of the compensated interferometer is greater than that of the Michelson interferometer for the same resolution by the factor $1 / n^{\prime}$. Thus, to achieve a given optical retardation (and therefore, a given spectral resolution), the instrument must be driven further than would have been the case with a conventional Michelson interferometer.

\section{FIELD-COMPENSATED INTERFEROMETERS}

Three interferometers have been built at Utah State University which employ prism compensators to increase the field of view. These interferometer-spectrometers have been used to obtain spectra of atmospheric gases by remote sensing. The first of these was engineered by Fon Brown and subsequently used at the University to obtain airglow measurements primarily in the visible portion of the spectrum. ${ }^{3}$

The second instrument was developed by Steed and Haycock and has been used to provide near infrared spectral measurements of airglow and auroral emissions as part of the ground support for rocket experiments. ${ }^{6}$ This instrument is cryogenically cooled and scans the 1 to $3 \mu \mathrm{m}$ spectral region at a resolution of $2 \mathrm{~cm}^{-1}$ with a scanning rate which may be as great as 10 scans per second. The optical throughput of this instrument is about $0.7 \mathrm{~cm}^{2} \mathrm{sr}$.

The third field-widened interferometer developed at the University was a rocketborne instrument engineered by Haycock ${ }^{4}$ and Steed. ${ }^{6}$ This instrument, pictured in Fig. 3, uses liquid nitrogen cooled calcium fluoride optics and a liquid helium cooled bismuth doped silicon infrared detector to cover the wavelength range from 2 to $8 \mu \mathrm{m}$. The

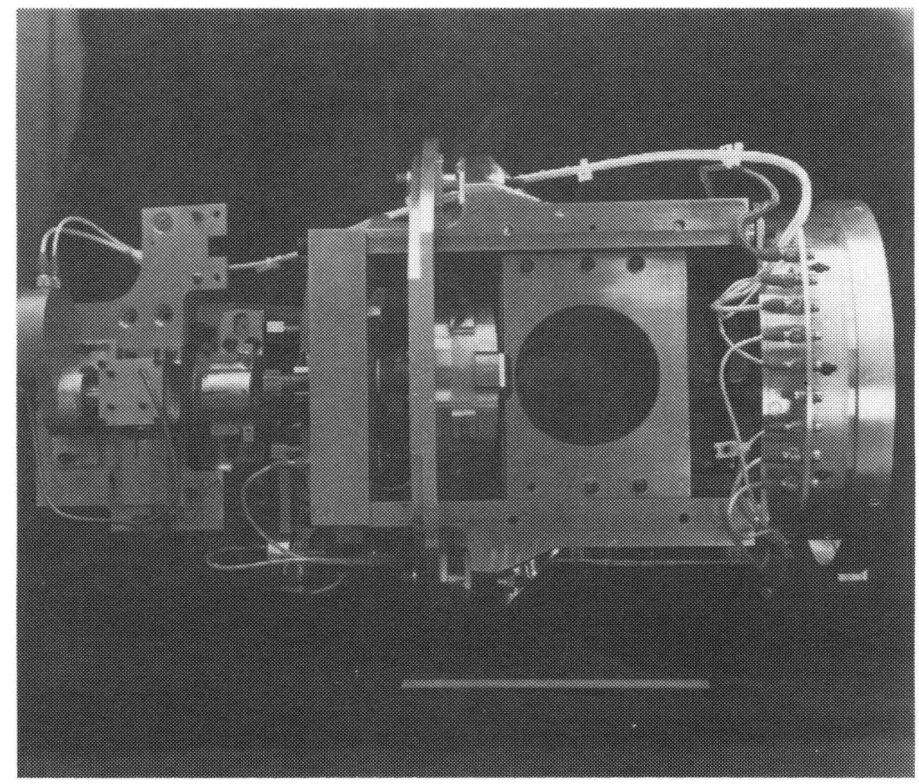

Fig. 3. Photograph of field-compensated interferometer developed by Utah State University for rocketborne environmental measurements.

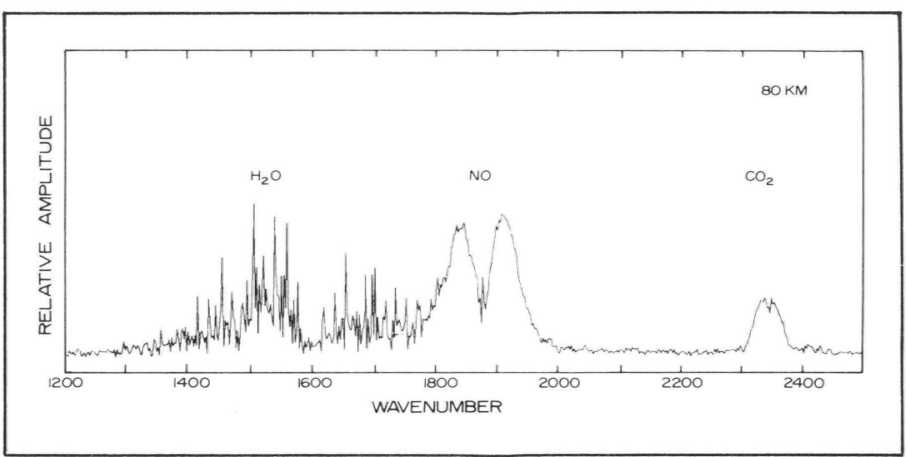

Fig. 4. Spectrum of zenith atmospheric near-infrared emissions taken aboard a rocket at $80 \mathrm{~km}$ using a field-compensated interferometerspectrometer.

resolution of the interferometer is $2.7 \mathrm{~cm}^{-1}$ and it has a scanning rate of one scan per 1.3 seconds.

Using calcium fluoride compensating prisms with 8 degree wedge angles, a field widening of 10.5 degrees full angle was achieved. The result was an instrument throughput of about $0.5 \mathrm{~cm}^{2} \mathrm{sr}$. This gave a noise equivalent spectral radiance (NESR) of $1 \times 10^{-12}$ watts $\mathrm{cm}^{-2} \mathrm{sr}^{-1}$ at $5 \mu \mathrm{m}$ for the spectrometer. To achieve this same NESR a conventional Michelson interferometer would require a scan duration of 80 seconds. This would have been impractical on a rocket flight. In general, the gain $\mathrm{g}$ of observation time shortening for a field-compensated relative to an uncompensated Michelson interferometer is ${ }^{1}$ 
$g=R^{2} \theta^{4}$

where $\theta$ (in radians) is the most oblique compensated ray.

\section{EXPERIMENTAL RESULTS}

Figure 4 shows a spectrum obtained from the field-of-view compensated interferometer during its flight aboard a rocket. The instrument was carried aboard Astrobee F No. A31.702, launched at White Sands, New Mexico, on August 3, 1979, at 9:20 hours Greenwich mean time. A pop cover which protected the instrument was opened at a rocket altitude of $79 \mathrm{~km}$ and the interferometer received light from the zenith as the rocket traveled on up to an apogee of $171 \mathrm{~km}$.

The spectrum of Fig. 4 was taken while the rocket was at an altitude of $80 \mathrm{~km}$. Emissions from the following atmospheric gases are clearly distinguishable from left to right: (1) water vapor, (2) nitric oxide and (3) carbon dioxide. The NO fundamental band at $1875 \mathrm{~cm}^{-1}(5.3 \mu \mathrm{m})$ is a very important high-altitude constituent of the atmosphere which is not observable from the ground.

\section{ACKNOWLEDGMENTS.}

The author wishes to acknowledge the help and contributions of Dr. A. T. Stair, Jr., of the U.S. Air Force Geophysical Laboratory who spon- sored the research program under which the infrared field-widened interferometers were developed. The works of Professors James C. Ulwick, Allan J. Steed, and Ralph H. Haycock of Utah State University are also recognized and greatly appreciated. Thanks also goes to Gene $A$. Ware for the assistance given.

\section{REFERENCES}

1. Doran Baker, "Field-Widened Interferometers for Fourier Spectroscopy," Spectrometric Techniques, Academic Press, New York, 71-106(1977).

2. P. Bouchareine and P. Connes, J. Phys. Radium 24, 134(1963).

3. A. M. Despain, F. R. Brown, Jr., A. J. Steed and D. J. Baker, "A LargeAperture Field-Widened Interferometer-Spectrometer for Airglow Studies," Aspen International Conference on Fourier Spectroscopy, A. F. Cambridge Research Laboratories, Bedford, Mass. 293-300(1971).

4. Ralph H. Haycock, "Rocket-borne Cryogenically Cooled Field-Widened Interferometer for the 2 to $8 \mu \mathrm{m}$ Spectral Region," Multiplex and/or High Throughput Spectroscopy, SPIE Vol 191, 120-129(1979).

5. Allan J. Steed, "Field-Widened Interferometry at Utah State Univeristy (USU)," Multiplex and/or High-Throughput Spectroscopy, SPIE Vol. 191, 2-14(1979).

6. A. J. Steed and D. J. Baker, "Night Sky Spectral Emission Measurements $(\lambda 0.9-2.3 \mu \mathrm{m})$ Using a Field-Widened Interferometer-Spectrometer," App. Optics 18, 3386-3389(1979).

7. Gene A. Ware, OH Rotational Temperatures Using Optimal Interferometric Techniques, Dissertation, Utah State University, Logan; 1980. 